\title{
SDRE and LQR Controls Comparison Applied in High-Performance Aircraft in a Longitudinal Flight
}

\author{
Guilherme P. Dos Santos a,1, Adriano Kossoski b,2, Jose M. Balthazar b,3, Angelo M. Tusset a, , $^{\star}$ \\ ${ }^{a}$ Federal University of Technology - Paraná (UTFPR), 84016-210, Ponta Grossa, PR, Brazil \\ b São Paulo State University, School of Engineering, 17033-360, Bauru, SP, Brazil \\ ${ }^{1}$ guilherme_aza@hotmail.com; ${ }^{2}$ adrianoutf@gmail.com; ${ }^{3}$ jmbaltha@gmail.com; ${ }^{4}$ a.m.tusset@ gmail.com* \\ * Corresponding Author
}

ARTICLE INFO

Article history

Received 03 May 2021

Revised 24 May 2021

Accepted 26 May 2021

Keywords

High-performance aircraft;

LQR control;

SDRE control

\begin{abstract}
This paper presents the design of the LQR (Linear Quadratic Regulator) and SDRE (State-Dependent Riccati Equation) controllers for the flight control of the F-8 Crusader aircraft considering the nonlinear model of longitudinal movement of the aircraft. Numerical results and analysis demonstrate that the designed controllers can lead to significant improvements in the aircraft's performance, ensuring stability in a large range of attack angle situations. When applied in flight conditions with an angle of attack above the stall situation and influenced by the gust model, it was demonstrated that the LQR and SDRE controllers were able to smooth the flight response maintaining conditions in balance for an angle of attack up to $56 \%$ above stall angle. However, for even more difficult situations, with angles of attack up to $76 \%$ above the stall angle, only the SDRE controller proved to be efficient and reliable in recovering the aircraft to its stable flight configuration.
\end{abstract}

This is an open-access article under the CC-BY-SA license.

\section{Introduction}

The dynamics analysis of aircraft models presents a great relevance considering the technological and scientific development of the last years, with new approaches and control designs being proposed to describe and improve the dynamics, control, and stability of the aircraft. In this context, fighter aircraft in combat situations have a critical behavior due to this system operating closer to their limit regions and dealing with higher speeds and a large variety of attack angles. For [1], the dynamics of an aircraft are naturally nonlinear due to the many forces acting on the system, such as drag and lift forces and the orientation of the air layer and its relationship with the chosen reference to describe it. Consequently, disregarding nonlinear aspects can be a limiting factor in the capabilities of the representative model of the system and consequently of its electronic controllers. According to [2], for a more realistic model, the nonlinearities and uncertainties intrinsically present must be considered to avoid unstable regions of operation, thus enabling more efficient and realistic control projects. 
Control approaches related to aircraft and autopilot are presented in different works, such as, [3] that have considered adaptive neural networks in flight control and [4] using optimal linear control in nonlinear longitudinal flight. This strategy can also be observed on [5] with nonlinear automatic flight control. Approaches developed by [6] with robust probabilistic control showed an increase in control performance. Considering current approaches with adaptative control proposes, such as the proposal by [7], with a multivariate allocation of adaptive control based on the spline to compensate for aerodynamic uncertainties, or also, proposed by [8] where we have an adaptive control based on Lyapunov function for longitudinal dynamics. Still with [9], is presented an autopilot project using loop modeling Ho for missiles with high attack angles. Finally, as addressed by [10], performing the nonlinear control project considering the longitudinal dynamics of a high-performance aircraft.

A wide variety of linear and nonlinear control approaches are proposed to meet the new demands for autopilot aircraft control in the literature. In [11], a PID controller is designed for the flight system of a helicopter and a quadcopter, where considerations are made regarding variations in the payload of the vehicles. In [12], the PID and Fuzzy control techniques are used to control an aircraft's pitch angle. Numerical results showed that both strategies were sufficiently good to satisfy the control design requirements. In [13], a PID controller is used to track the pitch angle of a mini aerial vehicle. Numerical results showed the efficiency of the proposed control method. In [14], the PID and the LQR with integral action control techniques were projected and compared to control the aircraft's pitch angle. Numerical results showed a better performance index for the PID control, while the LQR with integral action showed to be more suitable for eliminating steady-state errors.

In [15], the main subject is the lateral and longitudinal control of a fixed-wing Micro Air Vehicle (MAV). For this end, both a PID and an LQR with integral action control techniques were projected and compared. Results obtained by numerical simulations showed that both control strategies are efficient and robust in the presence of disturbances. In [16], a PID technique is designed to control an unmanned micro aerial vehicle. Numerical results have shown that the proposed control is efficient in controlling miniature aerial vehicles, but they lose performance in the presence of dynamics variations. In [17], the automatic control of the pitch angle of an aircraft is proposed using two different controllers: a classical PID and a heuristic method optimized I-PD. Numerical results demonstrated that the optimized I-PD control showed better results, especially for the transient region.

A Fuzzy controller using the Mandani rules is considered in [18] to control an aircraft pitch angle. A PID controller is designed in [19] to control the stability and performance of an aircrafttype system. The numerical results showed that the control gains obtained through the ZeiglerNichols method presented better flight stability and performance in a general manner for the studied case. In [20], the authors considered a PID controller with the gains optimized by a heuristic method to control the pitch angle of a large aircraft system with uncertainties. In [21], an extensive study on dynamics and flight control is presented, including demonstrating some codes used in both Matlab and Fortran languages.

In [22], a nonlinear control using an adaptive backstepping approach is considered to control the longitudinal dynamics of an unmanned aerial vehicle. Numerical results showed that the proposed control is efficient in controlling the aerodynamic velocity and the angle of flight through direct control over the deflections of the elevator and the thrust of the aircraft. An adaptive sliding mode control is considered in [23] for a mathematical model of a generic hypersonic vehicle subject to uncertainties and external disturbances. Simulation results demonstrated the robustness of the proposed control. In [24], a sliding mode control with integral action was proposed to stabilize a spacecraft attitude in the presence of external disturbances. Numerical results have shown that the proposed control is also robust in the presence of actuator failures. 
In [25] proposes a nonlinear dynamic inversion control for fighter aircraft. The data from the F16 aircraft is considered in the numerical simulations, and the results of the proposed control are compared with an adaptive integral sliding mode controller. In [26], an optimal Ho control is proposed for the control of the take-off and vertical landing of an underactuated aircraft. Numerical simulations for a nonlinear system demonstrated the efficiency of the proposed control. In [27], a robust $\mathrm{H}_{\infty}$ control is proposed for the path's tracking of a small, fixed-wing unmanned aircraft system. The dynamics of the aircraft were considered to be having six degrees of freedom, and the robustness of the controller was demonstrated by computer simulations. A proposal for automatic control of the aircraft's landing considering the longitudinal plane is presented in [28]. Errors and disturbances in the sensors are also considered. The combination of the $\mathrm{H}_{2}$ and $\mathrm{H}_{\infty}$ control techniques is proposed. The numerical results obtained are validated for the landing of a Boeing 747, and used to demonstrate the efficiency of the proposed control design.

In [29], it is proposed the control of the angle of flight for the longitudinal dynamics of an F16 aircraft. The proposed control is a robust nonlinear feedback control based on the sliding mode backstepping control technique. The robustness of the control is evaluated considering external disturbances, such as loading and unloading conditions during the flight. In [30], the adaptive control using feedback linearization is applied to an aircraft control system. Numerical simulations are presented to show that the proposed control is robust in the case of wind disturbance. In [31], a simple adaptive control with the implicit reference model is designed with the aim of ensuring smooth tracking of the rotation angle of the reference path, including the prevention of lateral oscillations. The results of numerical simulations demonstrate the efficiency of the proposed control strategy.

In [32], it is considered the application of an adaptive tracking control on aircraft wings. The proposed control is evaluated in a typical wing rock system, considering variations in the aerodynamic parameters. Numerical results show the efficiency of the proposed control, with excellent results for the transient regime. In [33], a dynamic analysis considering the singularities generated when an aircraft reaches critical speed is performed. Numerical simulations are considered to show the complexity of the system for wing morphing and considering different ranges of speeds around the critical one. Ref. [34] presents a robust analysis of a non-fragile control considering a delayed aircraft flight control system. The authors discuss the dissipative-based sampled data, using the Lyapunov stability theory and the Writinger-based inequality to solve the problem. A group of numerical simulations analyzing some fault situations are showed.

In [35], an incremental backstepping control system is designed for a nonlinear F/A-18 aircraft model. The results presented from numerical simulations showed that the proposed methodology is effective for flight control. In [36], it is proposed to control the flight path angle considering the exact state-space linearization methodology. The numerical results showed that the proposed control is effective for longitudinal flight. In [37], a nonlinear control is designed for flight control of a small unmanned aerial vehicle, including uncertainties and external disturbances in the system dynamics. Numerical results show that the proposed control is robust to satisfy the uncertainties and wind disturbances. In [38], a PID controller is proposed to improve the stability and performance of an aircraft system. The numerical results presented by the authors show that the gains obtained by the Zeigler-Nichols method provided good performance results, satisfying the design requirements.

The authors in [39] present an evolving neuro-fuzzy controller called PAC (Parsimonious Controller) for micro aerial vehicles considering the presence of a high level of environmental perturbations. The control was used in an over-actuated hexacopter and a bio-inspired flappingwing micro aerial vehicle. The authors presented numerical results about the performance of the controller. In [40], the authors propose the use of two high-order robust nonlinear 
controllers for controlling the maneuvers of a fighter aircraft in presence of uncertain parameters. The aileron, elevator, and rudder are controlled to change the output variables pitch, roll, and sideslip angles. Numerical simulations using a swept-wing type aircraft are presented. The final results presented by the authors show a great level of performance, where the aircraft was able to respond with bigger order longitudinal and lateral maneuvers, even in the presence of uncertainties.

As shown, there are several studies in the literature considering both linear and nonlinear dynamic models. It is of practical importance to use the linear control method as long as the nonlinear degree of the real system is not significant. However, if the nonlinear characteristics have a considerable impact on the analyzed operating condition, linear controllers will have a high chance of not fully meeting the design requirements [22, 41-44].

In this paper, two strategies for controlling the deflection of the elevator from an F-8 Crusader aircraft are designed and compared. The first one is the LQR (Linear Quadratic Regulator), a very common state-space linear control, and the second is the nonlinear SDRE (State-Dependent Riccati Equations) control. Both controls have the same objective: to recover the aircraft from the stall region caused by angles of attack greater than the stall angle, considering the aircraft's movement and its dynamic nonlinearities, as well as the effects of the variation of the aircraft speed in relation to the wind speed due to atmospheric turbulence.

\section{Mathematical Model for Longitudinal Flight of the F-8 Crusader Aircraft}

The dynamics of the F-8 Crusader aircraft are analyzed considering a mathematical model for flight dynamics, in which the forces and the coordinate system used to represent the movement of the aircraft are presented in Fig. 1. The drag force is neglected in relation to other parameters, and the moment of inertia is proportional to the total mass of the aircraft.



Fig. 1. The dynamic model of the aircraft

The lift force is divided into two components: wing and tail. The equations of the movement are developed in terms of four state variables $(x=(u, \alpha, \theta, q))$, where $u$ is the longitudinal flight speed, $\alpha$ represent the angle of attack, $\theta$ is the pitch angle, the aircraft's pitch rate is given by $q$ and $V$ is the variation in the wind speed (virtual wind tunnel) over time. 
The basic equations of longitudinal movement are represented in the system (1), where the drag was considered small enough to be disregarded compared to the thrust and weight [10].

$$
\left\{\begin{array}{c}
m(\dot{u}+w \dot{\theta})=-m g \sin \theta+L_{w} \sin \alpha+L_{t} \sin \alpha_{t} \\
m(\dot{w}-u \dot{\theta})=m g \cos \theta+L_{w} \cos \alpha+L_{t} \cos \alpha_{t} \\
I_{y} \ddot{\theta}=M_{w}+l L_{w} \cos \alpha-l L_{t} \cos \alpha_{t}-c \dot{\theta}
\end{array}\right.
$$

where $m=$ aircraft weight, $u=$ velocity of the aircraft in the $x$ direction, $w=$ velocity of the aircraft in the $z$ direction, $\theta=$ angular displacement around the $y$ axis, measured clockwise from the horizon as shown in Fig. 1, $g=$ constant of gravity, $I_{r}=$ moment of inertia of the aircraft around the $y$ axis, $L_{w}=$ wing lift, $L_{t}=$ tail lift, $\alpha=$ wing angle of attack, $\alpha_{t}=$ tail angle of attack, $\delta_{e}=$ deflexão do profundor, $M_{w}=$ wind moment, $l=$ distance between the aerodynamic center of the wing and the center of gravity of the aircraft, $l_{t}=$ distance between the aerodynamic center of the tail and the center of gravity of the aircraft, $c \dot{\theta}=$ damping moment.

The lift forces of the wing and tail are given by $L_{w}=C_{L_{w}} \bar{q} S$ and $L_{t}=C_{L_{t}} \bar{q} S_{t}$, respectively, where $C_{L}=$ wing lift coefficient, $C_{L_{t}}=$ tail lift coefficient, $\bar{q}=$ dynamic pressure, $S=$ wing area and $S_{t}=$ horizontal tail area. In addition, the moment of the wing $M_{w}$ is a function of $\alpha$, and the angle of attack of the aircraft's tail $\alpha_{t}$ is a function of both $\alpha$ and $\delta_{e}$.

Fig. 2 shows the vector representation of the velocity components $u, v$ and $w$ along the $x, y$ and $z$ axes [10].

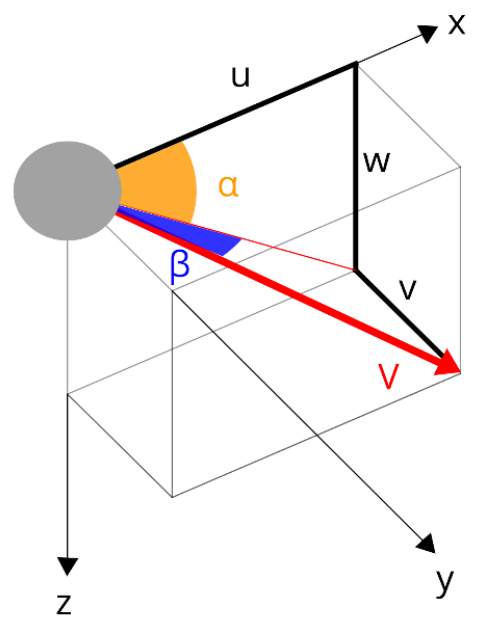

Fig. 2. Velocity components

Considering Fig. 2, one can define the following mathematical speed ratios [10] as

$$
\left\{\begin{array}{c}
w=u \tan \alpha \\
\dot{w}=\dot{u} \tan \alpha+u \dot{\alpha} \sec ^{2} \alpha
\end{array}\right.
$$

Replacing (2) in (1), one obtains

$$
\left\{\begin{array}{c}
\dot{u}=-u \dot{\theta} \tan \alpha-g \sin \theta+\left(L_{w} / m\right) \sin \alpha+\left(L_{t} / m\right) \sin \alpha_{t} \\
\dot{\alpha}=\dot{\theta} \sin ^{2} \alpha+(g / u) \sin \theta \sin \alpha \cos \alpha-\left(L_{w} / u m\right) \sin ^{2} \alpha \cos \alpha-\left(L_{t} / u m\right) \sin \alpha \cos \alpha \sin \alpha_{t}+ \\
\dot{\theta} \cos ^{2} \alpha+(g / u) \cos ^{2} \alpha \cos \theta-\left(L_{w} / u m\right) \cos ^{3} \alpha-\left(L_{t} / u m\right) \cos ^{2} \alpha \cos ^{2} \alpha_{t} \\
\ddot{\theta}=M_{w} / I_{y}+\left(l L_{w} / I_{y}\right) \cos \alpha-\left(l L_{t} / I_{y}\right) \cos \alpha_{t}-\left(c / I_{y}\right) \dot{\theta}
\end{array}\right.
$$

where the aircraft lift forces represented by $L_{w}$ and $L_{t}$ can be obtained using the following equations $[5,10]$ 


$$
\begin{gathered}
L_{w}=C_{L_{w}} \bar{q} S=\bar{q} S\left(C_{L_{w}}{ }^{0}+C_{L_{w}}{ }^{1} \alpha-C_{L_{w}}{ }^{2} \alpha^{3}\right)\left(1 /\left[1+(\alpha / 0.41)^{60}\right]\right) \\
L_{t}=C_{L_{t}} \bar{q} S_{t}=\bar{q} S_{t}\left(C_{L_{t}}{ }^{0}+C_{L_{t}}{ }^{1} \alpha_{t}-C_{L_{t}}{ }^{0} \alpha_{t}{ }^{3}+a_{e} \delta_{e}\right)
\end{gathered}
$$

where $C_{L_{w}}{ }^{0}, C_{L_{w}}{ }^{1}, C_{L_{w}}{ }^{2}, C_{L_{t}}{ }^{0}, C_{L_{t}}{ }^{1}$ e $C_{L_{t}}{ }^{2}$ are constants, $\delta_{e}$ represents the deflection angle of the horizontal tail measured at the right side of the $x$ axis and $a_{e}$ is the linear approximation of the effect defined by $\delta_{e}$ in $C_{L_{t}}$.

Substituting (4) and (5) in (3), and considering the following parameters for the F-8


$C_{m_{a . c}}=0 ; \bar{c}=3.53 \mathrm{~m} ; I_{y}=127512 \mathrm{Kgm}^{2} ; l=0.06 \mathrm{~m} ; l_{t}=5.01 \mathrm{~m}$. The aircraft's longitudinal motion equations are given by $[5,10]$

$$
\left\{\begin{array}{c}
\dot{u}=-u q \tan \alpha-10 \sin \theta+(\bar{q} / m)\left\{\begin{array}{c}
33.75 W \sin \alpha\left(4 \alpha-12 \alpha^{3}\right)+ \\
\left.+8.41 \sin \left(0.25 \alpha+\delta_{e}\right)\left[\begin{array}{c}
4\left(0.25 \alpha+\delta_{e}\right)- \\
-12\left(0.25 \alpha+\delta_{e}\right)^{3}+0.1 \delta_{e}
\end{array}\right]\right\}
\end{array}\right\} \\
\dot{\alpha}=q+(10 / u) \cos \alpha \cos (\alpha-\theta)-(\bar{q} / m u) \cos \alpha\left\{\begin{array}{l}
33.75 W\left(4 \alpha-12 \alpha^{3}\right)-8.41 \cos \left(0.75 \alpha+\delta_{e}\right) \\
{\left[4\left(0.25 \alpha+\delta_{e}\right)-12\left(0.25 \alpha+\delta_{e}\right)^{3}+0.1 \delta_{e}\right.}
\end{array}\right] \\
\dot{\theta}=q \\
\dot{q}=(50.1 / 127512) m \cos \theta-\left(171.1125\left(4 \alpha-12 \alpha^{3}\right) / 127512\right) \bar{q} W \cos \alpha-(50494.752 / 127512) q+ \\
+(\bar{q} / 127512)\left\{2.025\left(4 \alpha-12 \alpha^{3}\right) W \cos \alpha-42.1341 \cos \left(0.25 \alpha+\delta_{e}\right)\left[\begin{array}{c}
4\left(0.25 \alpha+\delta_{e}\right)- \\
-12\left(0.25 \alpha+\delta_{e}\right)^{3}+0.1 \delta_{e}
\end{array}\right]\right\}
\end{array}\right.
$$

Where $W=1 /\left(1+(\alpha / 0.41)^{60}\right)$.

To simulate the wind acting, two different contributions were used: one considered as a continuous action $\left(V_{0}\right)$, and another oscillating portion representing the gusts according to the following equation [10]

$$
\mathrm{V}(\mathrm{t})=V_{0}+\frac{1}{6}\left(\begin{array}{c}
10 \cos (1.57 t)+14 \cos (2.51 t)+18 \cos (3.77 t)+22 \cos (5.3 t) \\
+26 \cos (6.28 t)+30 \cos (7.48 t)
\end{array}\right)
$$

The dynamic pressure can be obtained by the following equation

$$
\bar{q}=\frac{1}{2} \rho V(t)^{2}
$$

\subsection{Numerical Simulations}

The following parameters will be considered for the numerical simulations: aircraft speed $\mathrm{V}_{0}=277.7[\mathrm{~m} / \mathrm{s}]$, the initial mass of the aircraft $m=9773[\mathrm{Kg}]$, atmospheric density at 9144 meters of altitude $\rho=0.4938$, and $\delta_{\mathrm{e}}=-0.1$ [rad]. The initial conditions are $\mathrm{u}_{0}=257.7[\mathrm{~m} / \mathrm{s}]$, $\alpha_{0}=0.22[\mathrm{rad}], \theta_{0}=0.21[\mathrm{rad}]$, and $\mathrm{q}_{0}=0 \mathrm{rad} / \mathrm{s}$.

Fig. 3 shows the time histories of the aircraft's longitudinal system represented by (6). According to the behavior shown in Fig. 3, it is possible to observe the variation of the main states of the aircraft over time, implying a long response rate, or even the aircraft stall situation as seen in Fig. 3(c), which could damage some systems and even cause the aircraft failure. In this kind of situation, human intervention will be necessary to control the system thus, directly depending on the pilot's skill and experience. The automatic control of the stall angle, and consequently of the main angles of the aircraft, becomes an alternative to keep the aircraft in the course and safe, even in critical flight conditions, easier the pilot work and composing the aircraft control system with autonomous flight. 




(a)

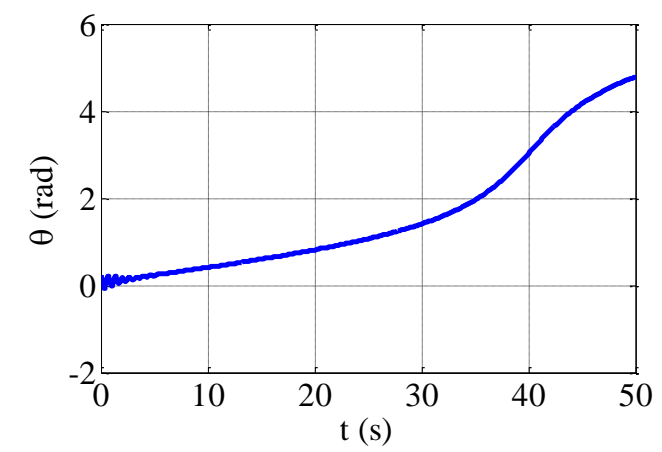

(c)



(b)

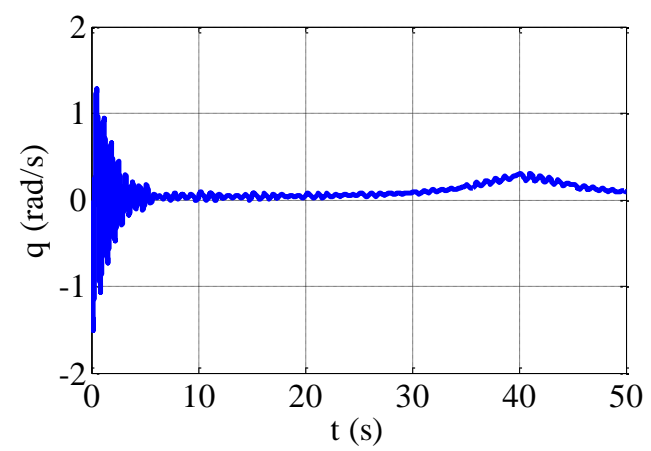

(d)

Fig. 3. Time histories of (a) aircraft's speed, (b) angle of attack of the aircraft, (c) aircraft's pitch angle, (d) aircraft's rate of the pitch.

\section{Proposed control}

The two control strategies used in the paper consider the introduction of a feedback control signal $(\boldsymbol{U})$ in the system (6).

Considering the system (6) in the matrix form with the feedback control [34]

$$
\dot{X}=A X+B U
$$

where $\boldsymbol{A}$ is the state matrix, $\boldsymbol{B}$ is the control matrix, and $\boldsymbol{U}$ is the feedback control, and it is defined as follows

$$
\boldsymbol{U}=-\boldsymbol{R}^{-1} \boldsymbol{B}^{T} \boldsymbol{P e}
$$

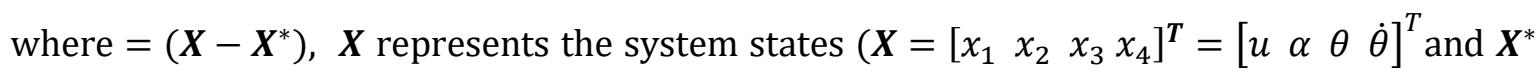
the desired states. Function $\boldsymbol{P}$ is obtained by solving the Ricatti equation defined as follows:

$$
\boldsymbol{P A}+\boldsymbol{A}^{T} \boldsymbol{P}-\boldsymbol{P B} \boldsymbol{R}^{-1} \boldsymbol{B}^{T} \boldsymbol{P}+\boldsymbol{Q}=\mathbf{0}
$$

The quadratic cost performance for the feedback control problem is given by

$$
J=\frac{1}{2} \int_{0}^{\infty}\left(\boldsymbol{e}^{T} \boldsymbol{Q} \boldsymbol{e}+\boldsymbol{U}^{T} \boldsymbol{R} \boldsymbol{U}\right) d t
$$

where $\boldsymbol{Q}$ and $\boldsymbol{R}$ are positive definite matrices.

Another important factor to consider is that the matrix cannot violate the system's controllability. The controllability is given by:

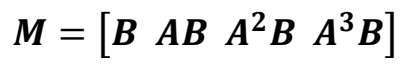

The system (6) is controllable if the rank of the matrix $\boldsymbol{A}$ is 4 . Control (10) will be used to control the system (6), considering $\boldsymbol{U}=\delta_{e}$. 


\subsection{Feedback Control by the LQR Control}

To obtain the control signal $\boldsymbol{U}$ considering the LQR control, the equation (6) is linearized, the $\boldsymbol{A}$ and $\boldsymbol{B}$ matrices are considered as follows

$$
\boldsymbol{A}=\left[\begin{array}{cccc}
0 & 0 & -10 & 0 \\
0 & 0 & 0 & 1 \\
0 & 0 & 0 & 1 \\
0 & -0.000331 \bar{q} & 0 & -0.396
\end{array}\right] \text { and } \boldsymbol{B}=\left[\begin{array}{c}
0 \\
34.481 \\
0 \\
-0.001354 \bar{q}
\end{array}\right] .
$$

Defining the $\boldsymbol{X}^{*}, \boldsymbol{Q}$ and $\boldsymbol{R}$ matrices as

$$
\boldsymbol{X}^{*}=\left[\begin{array}{c}
x_{1} \\
0.045 \\
x_{3} \\
x_{4}
\end{array}\right], \boldsymbol{Q}=\left[\begin{array}{cccc}
100 & 10 & 0 & 0 \\
10 & 1000 & 0 & 0 \\
0 & 0 & 1 & 0 \\
0 & 0 & 0 & 1
\end{array}\right] \text { and } R=[10000]
$$

Solving the Ricatti equation in (11), we obtain the following control signal $\boldsymbol{U}$ as

$$
\boldsymbol{U}=-0.1 x_{1}-0.2742\left(x_{2}-0.04\right)+0.7477 x_{3}+0.2625 x_{4}
$$

\subsection{Feedback control by the SDRE Control}

To obtain the control $\boldsymbol{U}$ considering the SDRE technique, one considers the A matrix as dependent on states $\boldsymbol{X}$ and $\mathbf{B}$ in the following linear form

$$
\boldsymbol{A}=\left[\begin{array}{cccc}
0 & x_{1} x_{4} & -10 & 0 \\
10 / x_{1}{ }^{2} & 8.41 \bar{q} / m x_{1} & 10 x_{2} / x_{1} & 1 \\
0 & 0 & 0 & 1 \\
0 & -0.000331 \bar{q} & 0 & -0.396
\end{array}\right] \text { and } \boldsymbol{B}=\left[\begin{array}{c}
0 \\
34.481 \\
0 \\
-0.001354 \bar{q}
\end{array}\right]
$$

And defining the $\boldsymbol{X}^{*}, \boldsymbol{Q}$ and $\boldsymbol{R}$ matrices as

$$
\boldsymbol{X}^{*}=\left[\begin{array}{c}
x_{1} \\
0.045 \\
x_{3} \\
x_{4}
\end{array}\right], \boldsymbol{Q}=\left[\begin{array}{cccc}
0.1 & 1 & 0 & 0 \\
1 & 10 & 0 & 0 \\
0 & 0 & 0.1 & 0 \\
0 & 0 & 0 & 0.1
\end{array}\right] \text { and } R=[1000] .
$$

As matrix $\mathbf{A}$, in this case, is dependent on the value of the states, the $\mathbf{U}$ control is also variable and depends on the states. Thus, a new control gain is obtained for each numerical integration of the system.

To obtain the $\boldsymbol{U}$ control signal through the SDRE control, the following algorithm must be adopted [41-44].

Step 1. Define the state-space matrix $\boldsymbol{A}$ with the state-dependent coefficients.

Step 2. Define $\boldsymbol{X}(0)=\mathbf{X}_{0}$, so that the rank of $\boldsymbol{M}$ is 4 and choose the coefficients of weight matrices $\mathbf{Q}$ and $\mathbf{R}$.

Step 3. Solve the Riccati equation in (11) for the state $\boldsymbol{X}$.

Step 4. Calculate the input signal from (10).

Step 5. Integrate of (6) and update the state of system $\boldsymbol{X}$ with the results.

Step 6. Calculate the rank of (13), if rank=4 return to step 4. If rank $<4$, continue using the last controllable matrix A obtained and return to step 4.

\subsection{Numerical Simulations}

For the numerical simulations of the proposed controls, the angle of attack will be varied with values above the stall angle to verify the efficiency and performance of the LQR and SDRE controllers applied in the recovery and stabilization of the aircraft during the flight. 
Considering this methodology, some parameters will have a fixed value, such as: $V_{0}=277.7$ $[\mathrm{m} / \mathrm{s}], m=9773[\mathrm{Kg}]$, and $\rho=0.4938$. Whereas the stall situation of the F-8 Crusader aircraft occurs at an angle of attack equals to 0.41 [rad] $\left(23.5^{\circ}\right.$ [deg]).

Fig. 4 shows the graphics for the speed, angle of attack, pitch angle and pitch rate, respectively. Considering the following parameters $u_{0}=257.7[\mathrm{~m} / \mathrm{s}], \alpha_{0}=0.62$ [rad], $\theta_{0}=0.61$ [rad], $q_{0}=0[\mathrm{rad} / \mathrm{s}], \delta_{\mathrm{e}}=-0.1[\mathrm{rad}]$ in the case without controllers and $\delta_{\mathrm{e}}=\mathrm{U}$ for the system with the linear LQR or nonlinear SDRE control.

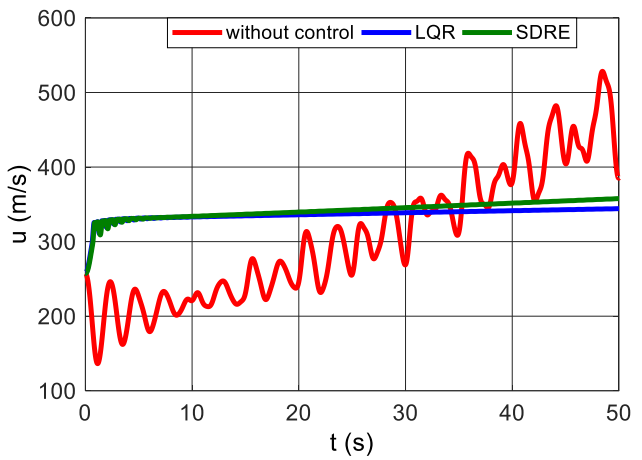

(a)



(c)

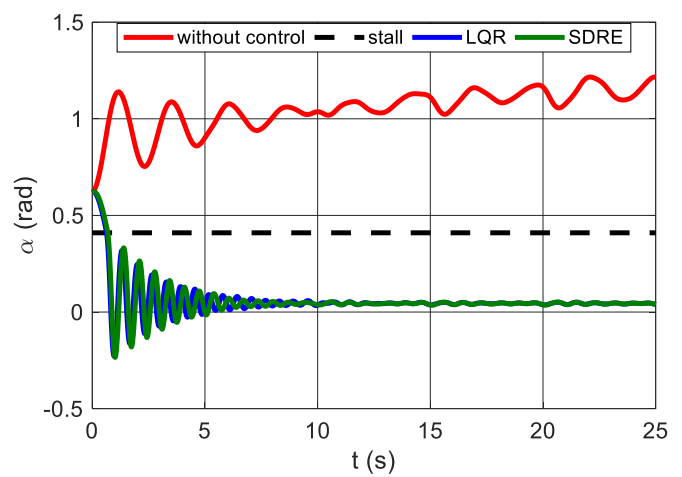

(b)



(d)

Fig. 4. Time histories of system with control (for $60 \%$ above the aircraft stall angle). (a) aircraft's speed, (b) angle of attack of the aircraft, (c) aircraft's pitch angle, (d) aircraft's rate of the pitch.

The aircraft speed behavior presented in Fig. 4(a) shows that the controllers have a critical impact on the answer of the system. This occurs because when subjected to an attack angle of $56 \%$ above the stall angle, the aircraft without the controller loses its ability to respond correctly to the forces suffered in this condition, where this situation can even cause a catastrophic failure. In both the situations with the LQR and SDRE controllers, similar speed behaviors are observed, with considerable stability compared with the uncontrolled system.

Fig. 4(b) shows the efficiency of the controllers in recovering the aircraft from an angle of attack of $\alpha=0.62$ [rad] ( $\left.35.6^{\circ}[\mathrm{deg}]\right)$, about $56 \%$ above the aircraft stall angle, which equals to 0.41 [rad] $\left(23.5^{\circ}\right.$ [deg]), in about 2 seconds. A relevant result since the recovery time of the aircraft must be fast, mainly for the cases in which the angle of attack is in some hostile regions of operation.

Analyzing Fig. 4(c) and Fig. 4(d), it is possible to observe the influence of the controllers on the aircraft dynamics, where the pitch angle refers to the angle of the aircraft. As shown in Fig. 4(b), the aircraft recovers from the stall situation, and as a consequence, the pitch angle is also controlled, this being shown in Fig. 4(c). The pitch rate demonstrates the rate of change in both 
the attack and pitch angles due to them being interconnected. Thus, observing the pitching rate, it is possible to define the speed at which the aircraft changes its main angles and consequently stabilizes or not its behavior. As can be seen in Fig. 4(d), the pitch rate is higher when the system is subjected to the action of the controllers, so the system tends to stabilize at a higher rate than when compared to the uncontrolled system. Considering the results presented in Fig. 4 , it can be concluded that the LQR and SDRE control proposed are efficient and present similar behavior in the aircraft's dynamic system, where both made it possible to recover the aircraft from situations up to $56 \%$ above the stall angle.

Fig. 5 shows the history of speed, angle of attack, pitch angle, and pitch rate, respectively, for cases above up to $80 \%$ of the aircraft stall angle. In this critical situation, the nonlinearities of the system are amplified as the angle of attack of the aircraft is increased. The system and controllers are also very influenced by these extreme stall regions. For the simulations, the following parameters are considered: $u_{0}=257.7[\mathrm{~m} / \mathrm{s}], \alpha_{0}=0.72[\mathrm{rad}], \theta_{0}=0.71$ [rad], $q_{0}=0$ $[\mathrm{rad} / \mathrm{s}]$ and $\delta_{\mathrm{e}}=-0.1[\mathrm{rad}]$ in case the system is not operating by the controllers and $\delta_{\mathrm{e}}=\mathrm{U}$ to the system with control.

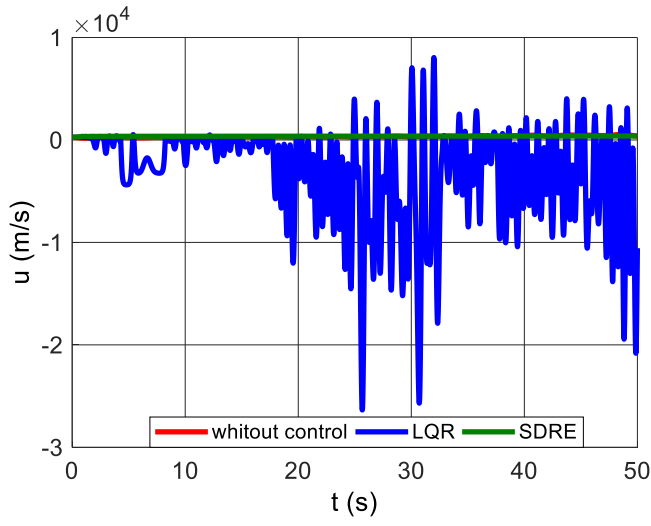

(a)

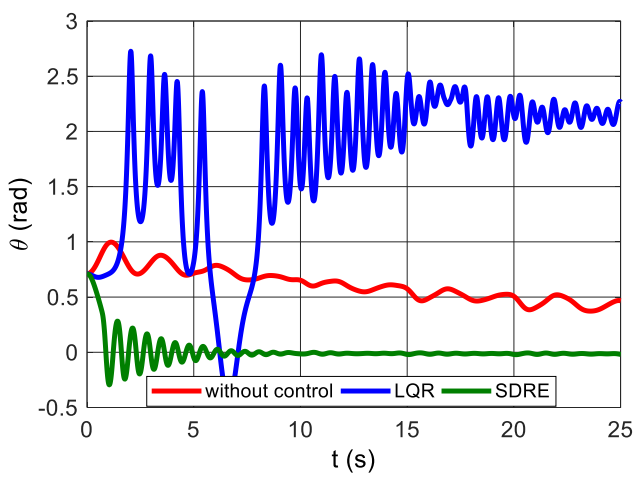

(c)



(b)

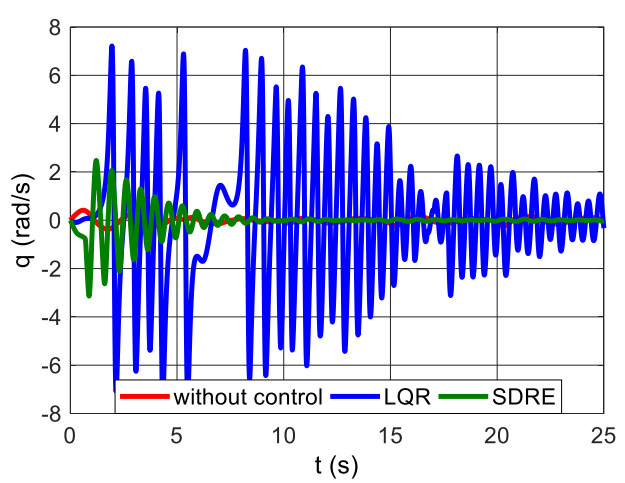

(d)

Fig. 5. Time histories of system with control (for $80 \%$ above the aircraft stall angle). (a) aircraft's speed, (b) angle of attack of the aircraft, (c) aircraft's pitch angle, (d) aircraft's rate of the pitch.

As shown in the results presented in Fig. 5(a), Fig. 5(c), and Fig. 5(d), only the SDRE control was efficient in controlling the aircraft's speed when the system was subjected to an angle of attack $76 \%$ above the stall angle. For Fig. 5b, we can observe the efficiency of the SDRE controller in recovering the aircraft from an angle of attack of $\alpha=0.72 \mathrm{rad}\left(41.3^{\circ} \mathrm{deg}\right)$, about $76 \%$ above the aircraft stall angle.

The results show poor performance for the LQR controller, which characterizes the incapability of the controller to act in such a way as to guarantee the stability of the aircraft for 
extreme cases, such as for situations of the angle of attack with $76 \%$ or more of the stall angle. The LQR and SDRE Controllers exhibit different behavior for this application region, demonstrating the reliability of the SDRE controller design and a limited application range for the LQR control design presented.

\section{Conclusion}

Considering the behavior of the aircraft shown in Fig. 4 and Fig. 5, it can be concluded that the SDRE controller presents an excellent performance when recovering the aircraft from the most critical stall situation that the system was submitted. As for the LQR controller, there is a limitation when the system is subjected to severe flight regions. For this aircraft and conditions analyzed, its application must be conditional or limited to situations with attack angles up to $56 \%$ above the stall angle. On the other side, the SDRE control could recover the system out of the stall region for angles of attack up to $76 \%$ above the stall angle for all the situations analyzed. Future work is considered the development of a mathematical model of the aircraft's flight dynamics with the lateral behaviors to increase the control project application.

\section{Acknowledgment}

The authors acknowledge the support from the Brazilian agencies CAPES, FA, and CNPq.

\section{References}

[1] B. Etkin and L. D. Reid, Dynamics of flight: stability and control. New York: Wiley, 1996.

[2] M. F. V. Pereira, I. A. Prado, D. F. De Castro, J. M. Balthazar, R. G. A. Da Silva and A. Nabarrete, "On Nonlinear Dynamics and Flight Control at High Angles of Attack with Uncertain Aerodynamics," In ASME 2016 International Mechanical Engineering Congress and Exposition, American Society of Mechanical Engineers, 2016, V04BT05A029. https://doi.org/10.1115/IMECE2016-67108

[3] A. J. Calise and R. T. Rysdyk, "Nonlinear adaptive flight control using neural networks," IEEE control systems, vol. 18, n. 6, 1998, pp. 14-25. https://doi.org/10.1109/37.736008

[4] D. C. Pereira, J. M. Balthazar, F. R. Chavarette, and M. Rafikov, "On nonlinear dynamics and an optimal control design to a longitudinal flight," Journal of Computational and Nonlinear Dynamics, vol. 3, no. 1, p. 011012, 2008. ttps://doi.org/10.1115/1.2802111

[5] W. L. Garrard and J. M. Jordan, "Design of Nonlinear Automatic Flight Control Systems," Automatica, Pergamon Press, vol. 13, no. 5, pp. 497-505, 1977. https://doi.org/10.1016/00051098(77)90070-X

[6] Q. Wang and R. F. Stengel, "Robust nonlinear flight control of a high-performance aircraft," Control Systems Technology, IEEE, vol. 13, no. 1, pp. 15-26, 2005, https://doi.org/10.1109/TCST.2004.833651

[7] H. J. Tol, C. C. Visser de, L. G. Sun, E. Kampen van and Q. P. Chu "Multivariate spline-based adaptive control of high-performance aircraft with aerodynamic uncertainties," Journal of Guidance and Control, vol. 39, no. 4, pp. 781-800, 2016. https://doi.org/10.2514/1.G001079

[8] F. Gavilan, R. Vazquez and J. A. Acosta, "Adaptive control for aircraft longitudinal dynamics with thrust saturation," Journal of Guidance, Control, and Dynamics, vol. 38, no. 4, pp. 651-661, 2015. https://doi.org/10.2514/1.G000028

[9] A. Mahmood, Y. Kim and J. Park, "Robust ho autopilot design for agile missile with time-varying parameters," Transactions on Aerospace and Electronic Systems, IEEE, vol. 50, no. 4, pp. 30823089, 2014. https://doi.org/10.1109/TAES.2014.130750

[10] G. P. Dos Santos, J. M. Balthazar, F. C. Janzen, R. T. Rocha, A. Nabarrete and A. M. Tusset, "Nonlinear dynamics and SDRE control applied to a high-performance aircraft in a longitudinal flight considering atmospheric turbulence in flight," Journal of Sound and Vibration, vol. 436, pp. 273285, 2018. https://doi.org/10.1016/j.jsv.2018.08.021 
[11] P. Pounds, D. R. Bersak and A. M. Dollar, "Stability of small-scale UAV helicopters and quadrotors with added payload mass under PID control," Aut Robots, vol. 33, pp. 129-142, 2012. https://doi.org/10.1007/s10514-012-9280-5

[12] Z. M. Motea, H. Wahid, J. Zahid, S. H. Lwin and A. M. Hassan, "A Comparative Analysis of Intelligent and PID Controllers for an Aircraft Pitch Control System," Applications of Modelling and Simulation, vol. 2, no. 1, pp. 17-25, 2018. http://arqiipubl.com/ojs/index.php/AMS_Journal/article/view/17

[13] R. V. K. Reddy, S. M. Sambasivam, S. Prem and C. Ramprasadh, "Design of a proportional-integral controller to track pitch angle in a mini aerial vehicle," International Journal of Micro Air Vehicles, vol. 9, no. 1, pp. 15-24, 2017. https://doi.org/10.1177/1756829316685181

[14] Y. Kanokmedhakul, N. Pholdee, S. Bureerat and N. Panagan, "LQR aircraft pitch controller design forhandling disturbance using differential evolution," Journal of Research and Applications in Mechanical Engineering, vol. 7, no. 2, pp. 145-153, 2019. https://ph01.tcithaijo.org/index.php/jrame/article/view/187455

[15] B. S. Anjali, A. Vivek and J. L. Nandagopal, "Simulation and analysis of integral LQR controller for inner control loop design of a fixed wing micro aerial vehicle (MAV)," Procedia Technology, vol. 25, 2016, pp. 76-83. https://doi.org/10.1016/j.protcy.2016.08.083

[16] X. Chen, E. U. Haq and J. Lin, "Design, modeling and tuning of modified PID controller for autopilot in MAVs," 2016 17th IEEE/ACIS International Conference on Software Engineering, Artificial Intelligence, Networking and Parallel/Distributed Computing (SNPD), 2016, pp. 475-480. https://doi.org/10.1109/SNPD.2016.7515943

[17] R. Monica and R. Monica, "Optimized Controller Structured to Solve Aircraft Pitch Control Problem," International Journal of Engineering and Technology, vol. 9, no. 2, pp. 1155-1162, 2017. https://doi.org/10.21817/ijet/2017/v9i2/170902281

[18] C. Johny and B. R. Varghese, "Pitch control of an aircraft using lead compensator \& fuzzy controller," International Journal of Creative Research Thoughts, vol. 6, no. 2, pp. 594-594, 2018. https://ijcrt.org/papers/IJCRT1892431.pdf

[19] G. Sudha and S. N. Deepa, "Opitimization for PID Control Parameters on Pitch Control of Aircraft Dynamics Based on Tuning Methods," Optimization for PID Control Parameters on Pitch Control of Aircraft Dynamics Based on Tuning Methods, vol. 10, no. 1, pp. 343-350, 2016. https://doi.org/10.18576/amis/100136

[20] K. P. Bharat and P. Sujatha, “QFT Robust Controller Design for Aircraft Pitch Control,” International Journal of Engineering and Management Research, vol. 7, no. 3, pp. 399-405, 2017. https://www.indianjournals.com/ijor.aspx?target=ijor:ijemr\&volume=7\&issue=3\&article=074

[21] B. L. Stevens, F. L. Lewis and E. N. Johnson, Aircraft control and simulation: dynamics, controls design, and autonomous systems. John Wiley \& Sons, 2015. https://doi.org/10.1002/9781119174882

[22] F. Gavilan, J. A. Acosta and R. Vazquez, "Control of the longitudinal flight dynamics of an UAV using adaptive backstepping," IFAC Proc, vol. 44, 2011, pp. 1892-1897. https://doi.org/10.3182/20110828-6-IT-1002.01876

[23] J. Guo, H. Zhang, X. Lu and J. Zhou, "Nonlinear disturbance observer-based adaptive sliding mode control for a generic hypersonic vehicle," International Journal of Aerospace Engineering, vol. 2018, p. 6978170, 2018. https://doi.org/10.1155/2018/6978170

[24] Q. Shen, D. Wang, S. Zhu and E. K. Poh, "Integral-type sliding mode fault-tolerant control for attitude stabilization of spacecraft," IEEE Transactions on Control Systems Technology, vol. 23, no. 3, pp. 1131-1138, 2015. https://doi.org/10.1109/TCST.2014.2354260

[25] S. Ijaz, C. Fuyang, M. T. Hamayun and H. Anwaar, "Adaptive integral-sliding-mode control strategy for maneuvering control of F16 aircraft subject to aerodynamic uncertainty," Applied Mathematics and Computation," vol. 402, 2021, p. 126053. https://doi.org/10.1016/j.amc.2021.126053

[26] K. D. Do, “Global inverse optimal control of vertical take-off and landing aircraft," IFAC Journal of Systems and Control, vol. 15, 2021, p. 100132. https://doi.org/10.1016/j.ifacsc.2020.100132

[27] D. Muniraj, M. C. Palframan, K. T.Guthrie and M. Farhood, "Path-following control of small fixedwing $\mathrm{H}_{\infty}$ unmanned aircraft systems with type performance," Control Engineering Practice, vol. 67, 2017, pp. 76-91. https://doi.org/10.1016/j.conengprac.2017.07.006 
[28] R. Lungu and M. Lungu, "Application of $\mathrm{H}_{2} / \mathrm{H}_{\infty}$ and dynamic inversion techniques to aircraft landing control," Aerospace Science and Technology, vol. 46, 2015, pp. 146-158. https://doi.org/10.1016/j.ast.2015.07.005

[29] V. H. Nguyen and T. T. Tran, "A Novel Hybrid Robust Control Design Method for F-16 Aircraft Longitudinal Dynamics," Mathematical Problems in Engineering, vol. 2020, 2020, p. 5281904. https://doi.org/10.1155/2020/5281904

[30] X. Yao and Y. Yang, "Adaptive Fault Compensation and Disturbance Suppression Design for Nonlinear Systems with an Aircraft Control Application," International Journal of Aerospace Engineering, vol. 2020, 2020, p. 4531302. https://doi.org/10.1155/2020/4531302

[31] B. Andrievsky, Elena V. Kudryashova, Nikolay V. Kuznetsov and O. A. Kuznetsova, "Aircraft wing rock oscillations suppression by simple adaptive control," Aerospace Science and Technology, vol. 105, 2020, p. 106049. https://doi.org/10.1016/j.ast.2020.106049

[32] R. Huang, J. Zhang and X. Zhang, "Adaptive tracking control of uncertain switched non-linear systems with application to aircraft wing rock," IET Control Theory \& Applications, vol. 10, no. 15, 2016, pp. 1755-1762. https://doi.org/10.1049/iet-cta.2015.1335

[33] J. Huang, X. Fu and Z. Jing, "Singular dynamics for morphing aircraft switching on the velocity boundary," Communications in Nonlinear Science and Numerical Simulation, vol. 95, p. 105625, 2021. https://doi.org/10.1016/j.cnsns.2020.105625

[34] Q. Zhu, S. V. Kumar, R. Raja and F. Rihan, "Extended dissipative analysis for aircraft flight control systems with random nonlinear actuator fault via non-fragile sampled-data control," Journal of the Franklin Institute, vol. 356, no. 15, 2019, pp. 8610-8624. https://doi.org/10.1016/j.jfranklin.2019.08.032

[35] L. Sun, L. Shi, W. Tan and X. Liu, "Flying qualities evaluation based nonlinear flight control law design method for aircraft," Aerospace Science and Technology, vol. 106, p. 106126, 2020. https://doi.org/10.1016/j.ast.2020.106126

[36] T. T. Tran and B. A. Newman, "Nonlinear flight control design for longitudinal dynamics," in: AIAA Guidance, Navigation, and Control Conference, Kissimmee, Florida, 2015, AIAA, 2015, pp. 1-16. https://doi.org/10.2514/6.2015-1994

[37] E. Safwat, W. Zhang, A. Kamel and M. Kassem, "Robustness Analysis of Modified Incremental Nonlinear Dynamic Inversion for Small UAVs," Automatic Control and Computer Sciences, vol. 54, pp. 128-138, 2020. https://doi.org/10.3103/S0146411620020078

[38] S. N. Deepa and G. Sudha, "Longitudinal control of aircraft dynamics based on optimization of PID parameters," Thermophysics and Aeromechanics, vol. 23, pp. 185-194, 2016. https://doi.org/10.1134/S0869864316020049

[39] M. M. Ferdaus, M. Pratama, S. G. Anavatti, M. A. Garratt, E. Lughofer "PAC: A novel self-adaptive neuro-fuzzy controller for micro aerial vehicles," Information Sciences, vol. 512, pp. 481-505, 2020. https://doi.org/10.1016/j.ins.2019.10.001

[40] K. Raj, V. Muthukumar, S. N. Singh, K. W. Lee, "Finite-time sliding mode and super-twisting control of fighter aircraft," Aerospace Science and Technology, vol. 82-83, pp. 487-498, 2018. https://doi.org/10.1016/j.ast.2018.09.028

[41] A. C. Alves, A. M. Tusset, J. M. Balthazar, J. J. Lima, F. C. Janzen, R. T. Rocha and A. Nabarrete, "SDRE Control Applied to the Wheel Speed of a Compressed Air Engine with Crank-Connecting-Rod Mechanism," Shock and Vibration, vol. 2017, pp. 1-14, 2017. https://doi.org/10.1155/2017/8340510

[42] A. M. Tusset, V. Piccirillo, A. M. Bueno, J. M. Balthazar, D. Sado, J. L. P. Felix, R. M. L. R F Brasil, "Chaos control and sensitivity analysis of a double pendulum arm excited by an RLC circuit based nonlinear shaker," Journal of Vibration and Control, vol. 22, 2016, pp. 3621-3637. https://doi.org/10.1177/1077546314564782

[43] J. J. Lima, A. M. Tusset, F. C. Janzen, V. Piccirillo, C. B. Nascimento, J. M. Balthazar, M. R. L. F. Brasil, "SDRE applied to position and vibration control of a robot manipulator with a flexible link," Journal of Theoretical and Applied Mechanics, vol. 54, pp. 1067-1078, 2016. https://doi.org/10.15632/jtam-pl.54.4.1067 
[44] D. R. Santo, J. M. Balthazar, A. M, Tusset, V. Piccirilo, R. M. L. R. F. Brasil, M. Silveira, "On nonlinear horizontal dynamics and vibrations control for high-speed elevators," Journal of Vibration and Control, vol. 24, pp. 825-838, 2018. https://doi.org/10.1177/1077546316667324 